\title{
Journal of Applied and Theoretical Physics Research
}

\section{Fullerenes of the $\Delta n=6$ series}

\author{
Alexander I. Melker ${ }^{1^{*}}$, Tatiana V. Vorobyeva ${ }^{2}$ and Ruslan M. Zarafutdinov ${ }^{1}$ \\ ${ }^{1}$ Department of Mechanics and Control Processes \\ ${ }^{2}$ Department of Experimental Physics
}

\begin{abstract}
*Corresponding author: Alexander I. Melker, Department of Mechanics and Control Processes, Peter the Great St. Petersburg State Polytechnic University, Polytekhnicheskaya 29, 195251, St. Petersburg, Russian Federation; E mail: newton@imop.spbstu.ru
\end{abstract}

Article Type: Research, Submission Date: 15 December 2017, Accepted Date: 22 December 2017, Published Date: 29 January 2018.

Citation: Alexander I. Melker, Tatiana V. Vorobyeva and Ruslan M. Zarafutdinov (2018) Fullerenes of the $\Delta n=6$ series. J Apl Theol 2(1): 1-4. doi: https://doi.org/10.24218/jatpr.2018.13.

Copyright: ( 2018 Alexander I. Melker, et al. This is an open-access article distributed under the terms of the Creative Commons Attribution License, which permits unrestricted use, distribution, and reproduction in any medium, provided the original author and source are credited.

\section{Abstract}

Fusion reactions of clusters and cupola half-fullerenes $\mathrm{C}_{4}, \mathrm{C}_{6} \mathrm{C}_{7}$, $\mathrm{C}_{9}, \mathrm{C}_{10}, \mathrm{C}_{12}, \mathrm{C}_{15}, \mathrm{C}_{18}, \mathrm{C}_{20}$ and $\mathrm{C}_{24}$ with each other are considered on the basis of Arrhenius's postulate. It means that at first there forms an intermediate compound and only afterwards a usual chemical reaction is going on. The final structure of fullerenes is obtained with the help of geometric modeling and is optimized through the use of Avogadro package. In general, the fullerenes which tend to take the appearance of a perfect spheroid have lesser formation energy. However, in some case self-organization of unshared electron pairs prevails.

Keywords: Cluster, Cupola, Fullerene, Fusion Reaction, Energy, Modelling.

\section{Introduction}

Fullerenes can be imagined to grow by reacting with each other, similar to bubbles in a soap solution. In Melker AI [1] we considered this possibility using geometrical modeling for the family of fullerenes obtained from the reactions $\mathrm{C}_{10}+\mathrm{C}_{10}, \mathrm{C}_{12}+\mathrm{C}_{12}$, $\mathrm{C}_{16}+\mathrm{C}_{16}, \mathrm{C}_{20}+\mathrm{C}_{20}$ and $\mathrm{C}_{24}+\mathrm{C}_{24}$. In this case fullerenes $\mathrm{C}_{20}, \mathrm{C}_{24}$, $\mathrm{C}_{32}, \mathrm{C}_{40}$ and $\mathrm{C}_{48}$ can be formed; the structure periodicity being $\Delta \mathrm{n}=8$, where $\mathrm{n}$ is the number of carbon atoms. Later on we found that the fullerenes can create the $\Delta n=10, \Delta n=12$ and $\Delta n=14$ periodicities [2-4]. All these periodicities have one and the same main characteristic feature; the fullerene structure changes from threefold symmetry to sixfold through four and fivefold ones. Taking this feature as a basis for fullerene classification, we have suggested the periodic system for such fullerenes. It consists of horizontal series and vertical columns (groups). The horizontal series form the $\Delta \mathrm{n}$ periodicities considered, the vertical columns include the fullerenes of one and the same symmetry, the mass difference $\Delta \mathrm{m}$ for each column being equal to a double degree of symmetry. We suppose that this feature can be taken as a basis for rigorous fullerene classification in addition to the geometric classification developed for mini-fullerenes [5].

In addition to the series $\Delta \mathrm{n}=8, \Delta \mathrm{n}=10, \Delta \mathrm{n}=12$ and $\Delta \mathrm{n}=14$, the fullerene stricture of which was designed elsewhere, it is possible to incorporate into the system other series. The fullerene structure for the series $\Delta \mathrm{n}=16$ was also obtained through the use of geometric modeling, but the results are not published. Now we want to increase the number of fullerenes fitted our classification by adding the fullerenes of the structure periodicity $\Delta n=6$; their graphs being designed [6]. In this contribution we present the structure and energy of such fullerenes. Knowing their structure allows one to activate the fullerenes, including unknown previously, for farther investigations and use of their properties. We suppose that the fullerenes of one and the same group have similar properties.

\section{Fusion Reactions of Cupola Half Fullerenes}

In 1889 Svante August Arrhenius postulated that a chemical reaction goes in the following way. At first there forms some intermediate compound and only afterwards a usual chemical reaction is going on. For fullerenes this postulate can be written as follows $A+B \rightarrow(A B) \rightarrow C$. In Melker AI [1] we have developed an algorithm that has proved itself in predicting the growth of perfect fullerenes conserving an initial symmetry, so called the fusion reaction algorithm. Now consider the reaction for fullerenes of the $\Delta \mathrm{n}=6$ series.

\section{Reactions leading to mini-fullerenes $\mathrm{C}_{14}$}

In Figure 1 the atomic configurations corresponding to reaction $C_{7}+C_{7} \rightarrow\left(C_{7} C_{7}\right) \rightarrow C_{14}$ and $C_{4}+C_{10} \rightarrow\left(C_{4} C_{10}\right) \rightarrow C_{14}$ are shown. At first two molecules $\mathrm{C}_{7}$, or $\mathrm{C}_{4}$ and $\mathrm{C}_{10}$, are moving towards each other (Figure 1, $a$ and $d$ ). Then the boundary atoms (dark-red) interact with each other producing a compound (Figure 1, $b$ and 
e). During this process new covalent bonds (heavy red lines) are generated, distorted polyhedrons are formed; they relaxing into perfect polyhedrons (Figure 1, $c$ and $f$ ). In the first case, the atomic configuration consists of six squares and three hexagons so it can be termed a tetra -hexa $_{3}$-polyhedron. It should be emphasized that the hexagons have a conformation known for cyclic molecules as a boat [7], so the configuration resembles more an elementary nanotube than a fullerene. In the second case, the atomic configuration consists of three squares and six pentagons so it can be termed a tetra ${ }_{3}$-penta $_{6}$ polyhedron. It is a basic truncated bipyramid. This structure was designed [6] on the basis of graph theory.

\section{Reactions leading to mini-fullerenes $\mathrm{C}_{18}$}

Analogously consider the atomic configurations corresponding to reactions $C_{9}+C_{9} \rightarrow\left(C_{9} C_{9}\right) \rightarrow C_{18}$ and $C_{6}+C_{12} \rightarrow\left(C_{6} C_{12}\right) \rightarrow C_{18}$ . As before, we have two cases of joining (Figure $2 a, b, c$ and Figure $2, d, e, f)$. The first case results in the atomic configuration corresponding to a perfect polyhedron that consists of two equilateral triangles, six pentagons, and three hexagons, so it could be named a tri -penta $_{6}$-hexa 3 polyhedron; the hexagons having also a boat conformation. The configuration also resembles more a nanotube than a fullerene. In the second case an isomer of fullerene is obtained; it is an all-vertices truncated triangular bipyramid.
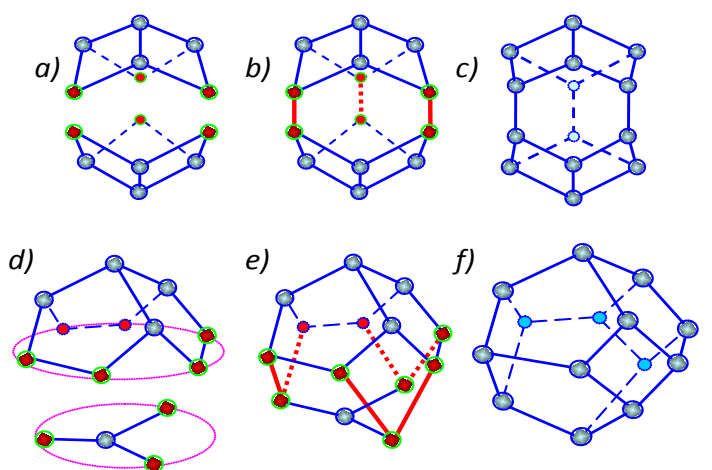

Figure 1: $\mathrm{C} 14$ as joining two cupolas $\mathrm{C} 7(a, b, c)$ and cluster $\mathrm{C} 4$ with cupola $\mathrm{C} 10(d, e, f)$; Dark-red and light-blue balls are reacting and neutral atoms, respectively; solid and dashed red lines are new covalent bonds a)
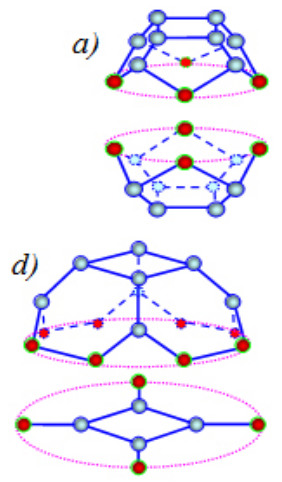
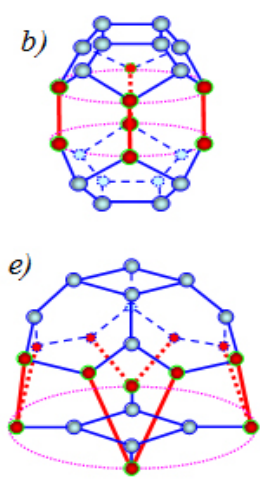

c)
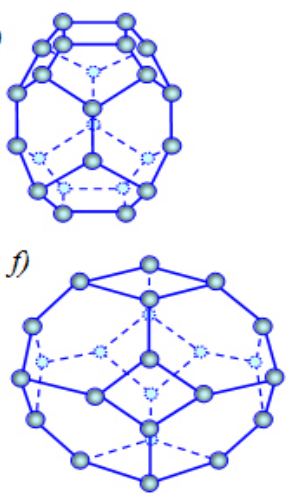

Figure 3: $\mathrm{C} 24$ as joining two cupolas $\mathrm{C} 12(a, b, c)$ and plane cluster $\mathrm{C} 8$ with cupola $\mathrm{C} 16(d, e, f)$; notations are the same as before

\section{Reactions leading to midi-fullerenes $\mathrm{C}_{24}$}

$C_{24}$ are $C_{12}+C_{12} \rightarrow\left(C_{12} C_{12}\right) \rightarrow C_{24}$ and $C_{8}+C_{16} \rightarrow\left(C_{8} C_{16}\right) \rightarrow C_{24}$ (Figure 3 ). The final atomic configuration in the first case is a perfect polyhedron that consists of two squares, eight pentagons, and four hexagons, i.e. a tetra - $^{-}$enta $_{8}-$ hexa $_{4}$ polyhedron; the hexagons having a boat conformation. In the second case an isomer of fullerene $\mathrm{C}_{24}$, a truncated octahedron (cuboctahedron), is obtained. It is worth noting that the cavities formed in f.c.c. metals by irradiation are also coboctahedra bounded by $\{100\}$ and $\{111\}$ planes [8]. As for fullerenes, four-membered rings have rarely been considered, but they are studied as the ends of narrow nanotubes [9].

\section{Reactions leading to midi-fullerenes $\mathrm{C}_{30}$}

$C_{30}$ can be written as $C_{15}+C_{15} \rightarrow\left(C_{15} C_{15}\right) \rightarrow C_{30}$ and $C_{10}+C_{20} \rightarrow\left(C_{10} C_{20}\right) \rightarrow C_{30}$. Their visualization is presented in Figure 4 . In the first case the final atomic configuration is a perfect polyhedron of twelve pentagons and five hexagons, so it could be named a penta ${ }_{12}$-hexa ${ }_{5}$ polyhedron; the hexagons having also a boat conformation. The five-membered rings creating cupolas are also studied as the ends of narrow nanotubes [9]. In the second case the fullerene obtained is a truncated pentagonal bipyramid. a)

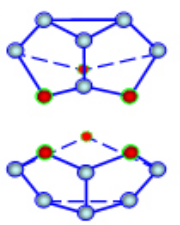

d)

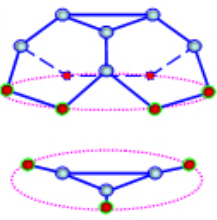

b)

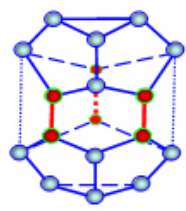

e)

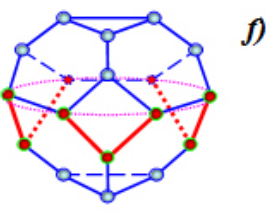

c)
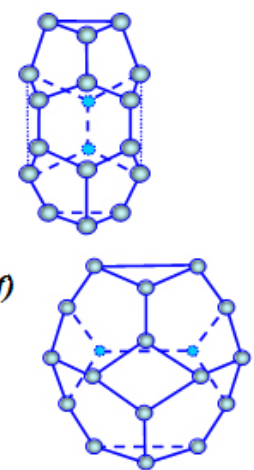

Figure 2: $\mathrm{C} 18$ as joining two cupolas $\mathrm{C} 9(a, b, c)$ and plane cluster $\mathrm{C} 6$ with cupola $\mathrm{C} 12(d, e, f)$; notations are the same as before
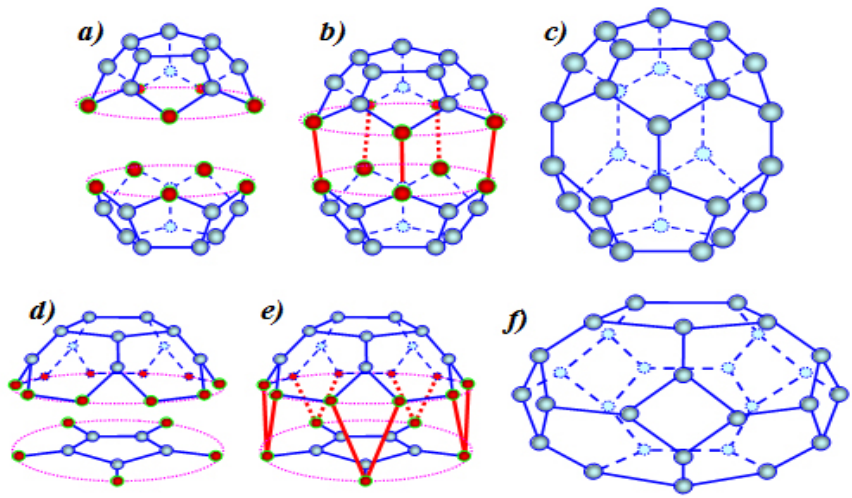

Figure 4: $\mathrm{C} 30$ as joining two cupolas $\mathrm{C} 15(a, b, c)$ and plane cluster $\mathrm{C} 10$ with cupola $\mathrm{C} 20(d, e, f)$; notations are the same as before 


\section{Reactions leading to midi-fullerenes $\mathrm{C}_{36}$}

$C_{36,}$ are $C_{18}+C_{18} \rightarrow\left(C_{18} C_{18}\right) \rightarrow C_{36}$ and $C_{12}+C_{24} \rightarrow\left(C_{12} C_{24}\right) \rightarrow C_{36}$ (Figure 5). In the first case the final atomic configuration is a perfect polyhedron of twelve pentagons and fourteen hexagons, so it could be named a penta ${ }_{12}$-hexa $_{14}$ polyhedron; the hexagons having also a boat conformation. In the second case it is a truncated hexagonal bipyramid. It should be mentioned that another way of forming a fullerene from a graphene flake, i.e. direct transformation of graphene to fullerene due to folding was considered [10].

\section{Single and Double Bonds, Energy}

We assume that the symmetry of double bonds location about the major axis of cupolas and fullerenes coincides with that of fullerene $\mathrm{C}_{60}$. Using this postulate, we have all the necessary input data for the optimization of the fullerene structures. The optimized structures of the fullerenes obtained through the use of Avogadro package [11] are shown in Figure 6. We have also calculated formation energies of these fullerenes (Figure 6). It should be emphasized that we developed a modified geometric graphics because the package graphics is incomprehensible.

The energy change for fullerenes can be explained in the following manner. According to the fourth of five basic empirical arguments [12], "geodesic structural factors should favor the more symmetric isomers, which can evenly disperse the strain from bond-angle deformation. This suggests that only 5/6-ring networks are likely to occur readily". From this it follows that the lesser is the fullerene surface, the lesser is its formation energy. Really, this is reflected in the energy of fullerenes $\mathrm{C}_{30}$ and $\mathrm{C}_{36}$ (above), but is invalid for $\mathrm{C}_{14}$ (above and below). We see that the rather low energy obtained unexpectedly contradicts to these arguments and needs more careful investigation. We suppose that in this case self-organization of unshared electron pairs (physical factor) [13] prevails over decreasing the ellipticity (mathematical point of view).
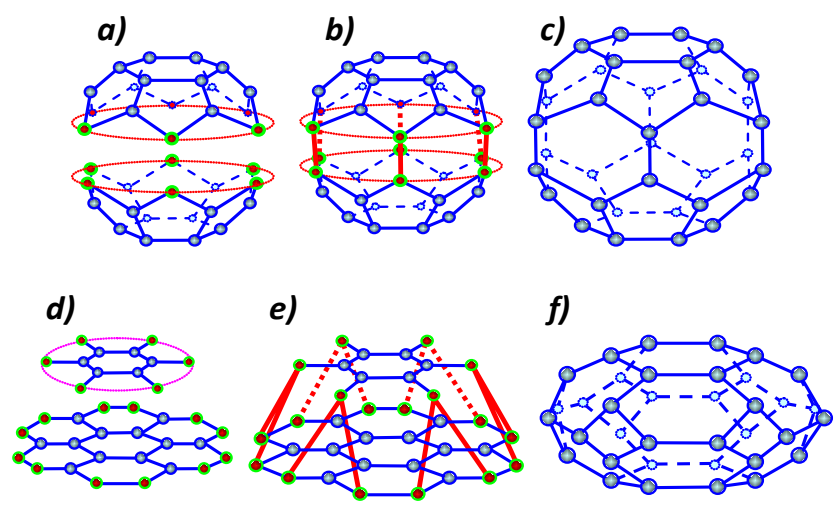

Figure 5: C36 as joining two cupolas $\mathrm{C} 18(\mathrm{a}, \mathrm{b}, \mathrm{c})$ and two graphene fragments $\mathrm{C} 12$ and $\mathrm{C} 24(\mathrm{~d}, \mathrm{e}, \mathrm{f})$; notations are the same as before

\section{Discussion}

Today there is no clear and unique theory of fullerene growth. "The problem here is not the lack of imagination, because quite numerous models have been proposed. What is rather lacking is a model using quantities that might be evaluated and measured. Moreover, a theoretical model, in order to deserve its name, should lead to numerical predictions. In order to represent something more than a set of circular arguments, a model should predict more numerical values, parameters or functional relations than the number of input parameters" [14].

At this time there are a lot of papers on fullerene properties [15, and 277 references therein]. Using different computational methods (there are also a lot of programs), the authors calculate the properties of the most popular fullerenes which structure is known. As a result, the numbers obtained contradict to each other and only increase disordered information. To our mind, the absence of appreciable progress in understanding fullerene nature is determined by the domination of numerical calculations on the known structures. However, such numerical calculations are unable to predict new structures, so the 'numerical progress' results mainly in increasing numerical entropy. It should be emphasized that numerical calculations are not a theory, but a kind of numerical experiment [16].

Consequently, it is necessary not to do calculations for the sake of calculations, but at first to develop a system (mathematical model) as a basis for the calculations. The first step of creating any mathematical model is the formulation of the laws that connect the main objects of the model [17]. In our case the main objects are perfect basic fullerenes; we have also the preliminary law in the form of the periodic system [3] and the fusion algorithm for obtaining perfect basic fullerenes, but we do not know the structure of all the main objects. Besides, not all perfect fullerenes, the structure of which being known, are incorporated in the suggested periodic system.

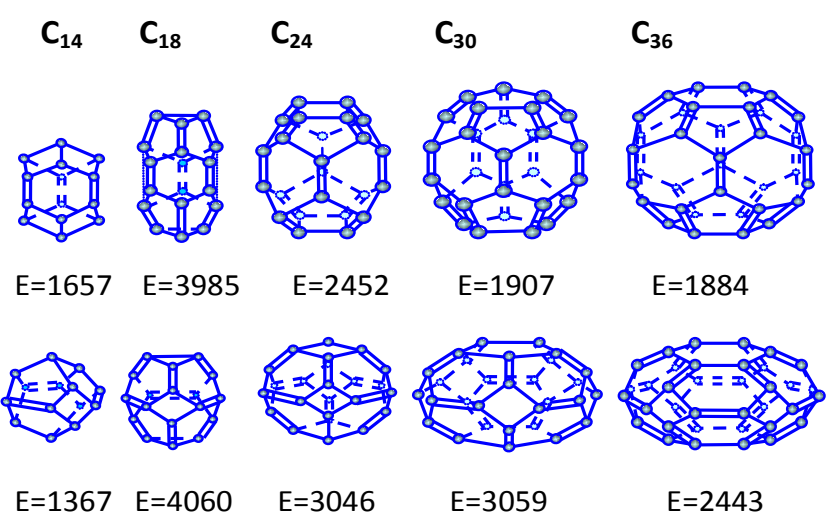

Figure 6: Structure and energy $(\mathrm{kJ} / \mathrm{mol})$ of the $\Delta \mathrm{n}=6$ series fullerenes with single and double bonds 
Therefore, the next step in our investigation is obtaining the structure and energy of missing fullerenes with the purpose to incorporate the missing known and unknown fullerenes in the periodic system. Only afterwards, having a comprehensive picture, it seems reasonable to try to explain why some fullerenes are more stable than others.

\section{References}

1. Melker Al, Vorobyeva TV. Fusion reactions of cupola halffullerenes. St. Petersburg State Polytechnical University Journal: Physics and Mathematics. 2016; 2(3):209-216. doi: https://doi. org/10.1016/j.spjpm.2016.07.002.

2. Melker Al, Krupina MA, Zarafutdinov RM. Structure and energy of the $\Delta n=10$ series fullerenes. Proceedings of NDTCS; 2017. 17(2017):68-71.

3. Melker Al, Krupina MA, Zarafutdinov RM. Periodic system for basic perfect fullerenes obtained by fusion reactions. Proceedings of NDTCS; 2017. 17(2017):72-75.

4. Melker Al, Vorobyeva TV, Zarafutdinov RM. Structure and energy of the $\Delta n=14$ series fullerenes. Proceedings of NDTCS; 2017. 17(2017):79-82.

5. Melker Al, Starovoitov SA, Vorobyeva TV. Classification of minifullerenes on graph basis. Materials Physics and Mechanics. 2014; 20(1):12-17.

6. Melker Al, Krupina MA. Designing mini-fullerenes and their relatives on graph basis. Materials Physics and Mechanics. 2014; 20(1):18-24.

7. Melker Al, Krupina MA. Hidden symmetry or why cyclic molecules have so strange forms. Materials Physics and Mechanics. 2010; 9(1):11-19.

8. Melker Al. Dynamics of Condensed Matter. Vol. 2. Collisions and Branchings. St. Petersburg Academy of Sciences on Strength Problems. 2010. p. 342.
9. Slanina $Z$, Zhao X, Uhlik F. Model narrow nanotubes related to $\mathrm{C}_{36^{\prime}}$ $\mathrm{C}_{32}$ and $\mathrm{C}_{20}$; initial computational structural sampling. Materials Science and Engineering. 2002; 96(1):164-168. doi: https://doi. org/10.1016/S0921-5107(02)00312-4.

10. Chuvilin A, Kaiser U, Bichoutskaia E, Besley N, Khlobystov A. Direct transformation of graphene to fullerene. Nat Chem. 2010; 2(6):450-453. doi: 10.1038/nchem. 644.

11. Hanwell MD, Curtis DE, Lonie DC, Vandermeersch DC, Zurek E, Hutchison GR. Avogadro: an advanced semantic chemical editor, visualization, and analysis platform. Journal of Cheminformatics. 2012; 4:17. doi: https://doi.org/10.1186/1758-2946-4-17.

12. Kroto HW. The stability of the fullerenes $C_{n}$, with $n=24,28,32,36$, 60, 60 and 70. Nature. 1987; 329:529-531.

13. Melker Al, Romanov SN, Kornilov DA. Computer simulation of formation of carbon fullerenes. Materials Physics and Mechanics. 2000; 2(1):42-50.

14. Kerner R. Nucleation and growth of fullerenes. Computational Materials Science. 1994; 2(3-4):500-508. doi: https://doi. org/10.1016/0927-0256(94)90080-9.

15. Irle S, Page AJ, Saha B, Wang Y, Chandrakumar KRS, Nishimoto Y, et al. Atomistic mechanisms of carbon nanostructure self-assembly as predicted by nonequilibrium QM/MD simulations. In: Practical Aspects of Computational Chemistry II: An Overview of the Last Two Decades and Current Trends. Leszczynski J, Shukla MK, editors. Springer-European Academy of Sciences; 2012. p. 59.

16. Melker Al. Modeling Experiment. Znanie, Moscow; 1991. p. 64.

17. Mathematical Encyclopedic Dictionary, Soviet Encyclopedia, Moscow; 1988. p. 848. 